\section{Sarcopenia, frailty and exercise}

Many thanks to Offord and Witham for their article on sarcopenia. ${ }^{1}$ They rightly identify low levels of physical exercise as a major cause of sarcopenia and subsequent frailty, dependency and premature death. As they say, exercise has some effect in reversing sarcopenia but by the time that sarcopenia has become a clinical problem it is probably too late to treat. A trial that investigated weight-training in nursing home residents, aged 90 years or older, showed weight training to be clearly beneficial in terms of strength and self-care scores but, the scores soon fell back to their pre-trial levels after the study ended. ${ }^{2}$ Prevention is what is needed and this means regular exercise for the population as a whole, starting as early in life as possible and continuing throughout life.

Regular moderate to vigorous exercise is not only a protection against sarcopenia but is also the key to prevention of a large number of non-communicable diseases which themselves lead to a further reduction of physical performance - obesity, diabetes, heart disease, osteoporosis, some cancers and many others. Regular exercise lengthens lifespan but, perhaps more importantly, healthspan. The compression of morbidity at the end of life is what we all seek for our patients as well as ourselves. It is regular exercise which lengthens healthspan and reduces the financial, social and health costs which result from an increasingly dependent elderly population. The desirability of decreasing the period of dependency at the end of life cannot be overstated.

Sadly it seems that end-of-life dependency is actually increasing. The Office of National Statistics report in 2012 showed that a 65 year old man could expect to be free from disability and long term illness for a further 10.6 years but by 2014 this had decreased to 10.3 years. For women the figures were 11.2 years falling to 10.9 years. ${ }^{3}$

Inactivity should be managed as seriously as cigarette smoking. Public education, the provision of better exercise facilities and the promotion of active travel (ie walking or cycling to work) should become political imperatives. And the medical profession should spend a great deal more time and effort in encouraging an idle population to get off its collective backside and start exercising.

HUGH JN BETHELL The Cardiac Rehabilitation Centre, Alton

\section{References}

1 Offord NJ, Witham MD. The emergence of sarcopenia as an important entity in older people. Clinical Medicine 2017;17:363-6.

2 Faber M, Bosscher R, Chin A, et al. Effects of exercise programs on falls and mobility in frail and prefrail older adults: a multicenter randomized controlled trial. Arch Phys Med Rehabil 2006;87:885-96.

3 Office of National Statistics. Disability-free life expectancy by upper tier local authority: England 2012 to 2014. Statistical Bulletin 2016.

\section{Antisynthetase syndrome}

Editor - Fallon et al report a typical case of antisynthetase syndrome (ASS) responsive to prednisolone and mycophenolate. ${ }^{1}$

While the association of the related autoimmune connective tissue disorder dermatomyositis with malignancy is well established, a link with the presence of antisynthetase antibodies and malignancy is only just emerging. A recent case series reported the development of malignancy in 8/124 patients. $^{2}$

Both conditions are routinely treated with potent immunosuppressive therapy which further increases this risk of malignancy.

Readers are reminded to monitor for malignancy in patients with antisynthetase syndrome in the same fashion as they currently do with patients with dermatomyositis particularly when using long term immunosuppressives such as mycophenolate or azathioprine.

GRAHAM JOHNSTON Consultant dermatologist and honorary associate professor, Leicester Royal Infirmary

\section{References}

1 Fallon J, Majeed S, Adamali H, Gunawardena H. Lesson of the month 2: Dry skin, yellow nails and breathlessness. Clin Med 2017;17:371-2.

2 Shi J, Li S, Yang $\mathrm{H}$ et al. Clinical profiles and prognosis of patients with distinct antisynthetase autoantibodies. J Rheumatol 2017;44:1051-7.

\section{The hyperacute neurology team}

Editor - I enjoyed reading the paper on a hyperacute neurology team ${ }^{1}$ which certainly seems to produce impressive results. I was left wondering however, with a consultant-delivered service, about their education and training responsibilities. Does the team have these? If so, how do they impact on the service? If not, how is their expertise transmitted to the next generation?

JOHN COOKSON

Retired physician

\section{Reference}

1 Nitkunan A, MacDonald B, Boodhoo A et al. A hyperacute neurology team - transforming emergency care. Clin Med 2017;17:298-302.

Editor - Nitkunan et al's ${ }^{1}$ interesting article outlines some innovative ideas to ensure that patients are promptly seen by neurologists after admission to a district general hospital. They highlight the role of an acute neurology nurse co-ordinator and the advantages of a service being based close to the acute medical unit.

Our district general hospital also provides a 5-day service, with patients being seen on the same day if referred before 2 $\mathrm{pm}$. There has been a similarly marked increase in number of referrals to the team. The number of referrals increased by $38 \%$ between the first six months of 2014 and the comparable period in 2015 , were static to 2016 , but increased by a further $22 \%$ in the first half of this year.

In response to this growing demand we introduced another individual who could be considered when constructing a hyperacute neurology team - a neurology clinical fellow. This would typically be an individual who has completed Core Medical Training and who is interested in obtaining further experience in neurology. They can provide an easily accessible 
liaison point with medical teams and can help provide prompt review even with rising referral numbers. Indeed, from January to July 2017, after introduction of the neurology clinical fellow, only $5.1 \%$ of patients were not seen on the day a referral was received. In contrast in 2015 and $201610.2 \%$ and 10.9\% reviews were delayed for referrals in the first half of the year.

For such a position to succeed considerable input from consultants is needed, particularly given the fellow's possible lack of experience in neurology. The consequences of inadequate supervision have been highlighted elsewhere. ${ }^{2}$ The fellow may not have the administrative skills of a neurology nurse co-ordinator but they bring other attributes.

Nitkunan et al found that siting their hyperacute neurology team close to the acute medical unit allowed an approachable referral system to be created. A hospital's layout may make this difficult, and we instead have a computerised referral system which also avoids a formal referral approach. Designing acute neurology care may require different solutions across the NHS.

\section{Conflicts of interest}

No conflicts of interest to declare.

DAVID WHITESIDE Neurology clinical fellow, Northwick Park Hospital

\section{References}

1 Nitkunan A, MacDonald B, Boodhoo A et al. A hyperacute neurology team - transforming emergency care. Clin Med 2017;17:298-302.

2 Yogarajah M, Mirfenderesky M, Ahmed T, Schon F. Consultant supervision of trainees seeing inpatient ward referrals - a cause for concern? Clin Med 2014;14:268-73.

\section{Response}

Editor - Both letters above raise issues about the place of nonconsultant staff.

We agree that different units will, no doubt, develop different models depending on their needs. It is key to collect and publish data with similar outcome measures so that genuine comparisons of efficacy can be made.

Whatever the layout of the hospital, almost all now have 'acute admission units' with acute physicians closely linked to emergency departments. That is where the person leading the acute neurology team should be based; in our case it is the acute neurology nurse.

The model we report is based on using exclusively senior staff: consultant neurologists, a band 8 nurse triaging patients and band 7 epilepsy nurses. Acute neurology requires two critical decisions:

$>$ which patients are safe for early discharge

$>$ what sort of follow up arrangements do these 'early discharge' patients require.

These decisions are difficult, require vast experience and are fraught with potential medico-legal pitfalls.

We strongly believe in providing both a high quality clinical service as well as training but that these two aspects require different approaches.

Supervision of trainees nationally is problematic as highlighted in our recent paper. ${ }^{1}$ In our experience supervising trainees properly requires great time and commitment. Neurology trainees are disproportionately based in tertiary centres but would benefit enormously from time in DGHs like ours where there is a major commitment to service and training. Approximately half of funding for trainees is derived from Health Education England via the Local Education and Training Board and half is from the trust itself. With neurology inpatient beds (and therefore income generated) at tertiary centres, it is difficult for DGHs to fund neurology juniors. Changes envisaged in the Shape of Training could feed into this debate.

ARANI NITKUNAN

BRIDGET K MACDONALD

AJAY BOODHOO

ANDREW TOMKINS

CAITLIN SMYTH

MEDINA SOUTHAM FRED SCHON

Croydon University Hospital, Croydon, UK

\section{Reference}

1 Yogarajah M, Mirfenderesky M, Ahmed T, Schon F. Consultant supervision of trainees seeing inpatient ward referrals - a cause for concern? Clin Med 2014;14:268-73. 